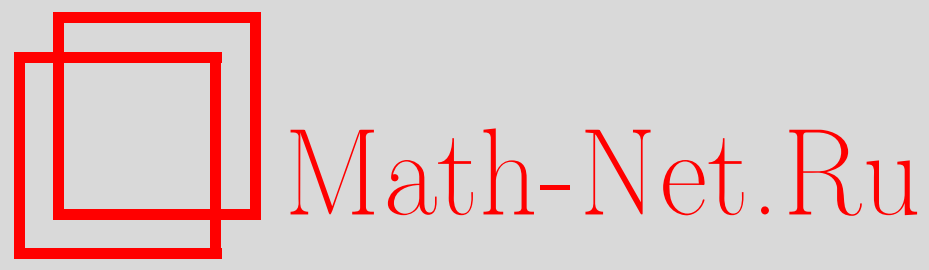

В. В. Вершинин, Э. Осса, Спектры Тома коммутантов обобщенных групп кос, Функи. анализ и его прил., 1998, том 32, выпуск 4, 1-9

DOI: https://doi.org/10.4213/faa435

Использование Общероссийского математического портала MathNet.Ru подразумевает, что вы прочитали и согласны с пользовательским соглашением

http://www . mathnet.ru/rus/agreement

Параметры загрузки:

IP: 54.197 .130 .99

26 апреля 2023 г., 14:18:21

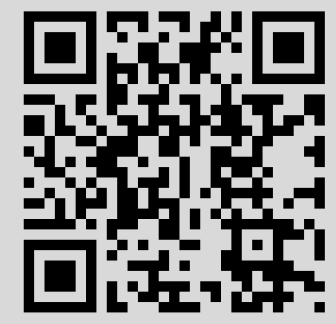


Функииональньй анализ и его приложения

1998, т. 32, вып. 4, с. 1-9

УДК 512.664+515.146.6

\title{
Спектры Тома коммутантов обобщенных групп кос ${ }^{\star}$
}

\author{
(c) 1998. В. В. ВеРшинин, Э. ОССА
}

\section{Введение}

Первым рассматривал когомологии групп кос В. И. Арнольд $[1,2]$. Изучение этих когомологий связано с различными математическими областями и задачами. Арнольд [2] применял их к проблеме представимости алгебраических функций нескольких переменных суперпозициями алгебраических функций от меньшего числа переменных. В. А. Васильев [3] и С. Смейл [4] использовали их для оценок сложности алгоритмов. М. Маховальд $[5,6]$ и Ф. Коэн [7], используя когомологии групп кос, доказали, что спектр Тома, порожденный серией групп кос, эквивалентен спектру Эйленберга-Маклейна, соответствующему обычным гомологиям с коэффициентами в $\mathbb{Z} / 2$. Связи с теорией конфигурационных пространств и пространств петель изучались П. Делинем [8], Г. Сигалом [9], Д. Б. Фуксом [10], Ф. Коэном [11], Э. Оссой [12] и другими. Полное вычисление этих когомологий с коэффициентами в $\mathbb{Z} / 2$ было проведено Фуксом [13]. Когомологии с коэффициентами в $\mathbb{Z}$ изучались Коэном [11] и Ф. В. Вайнштейном [14]. В. В. Горюнов $[15,16]$ выразил когомологии обобщенных групп кос серий $C$ и $D$ через гомологии классических групп кос. Рассматривались также когомологии подгрупп групп кос. Арнольд [17] вычислил когомологии групп крашеных кос, Коэн [18] и Э. В. Френкель [19] изучали когомологии коммутантов групп кос. В настоящей работе мы рассматриваем спектры Тома, порожденные коммутантами обобщенных групп кос. Интерес к таким спектрам вызван тем, что они связаны с целочисленными (или локализованными) гомологиями.

\section{$\S 1$. Основные определения и конструкции}

Пусть $B r_{n}$ обозначает группу кос из $n$ нитей $[20,21], \Sigma_{n}$ обозначает симметрическую группу, а $\tau_{n}$ - канонический эпиморфизм:

$$
\tau_{n}: B r_{n} \rightarrow \Sigma_{n}
$$

Предположим, что в евклидовом пространстве $\mathbb{R}^{n}$ действует конечная группа Кокстера $W_{n}[22]$. Группа $W_{n}$ порождена отражениями, и, следовательно, определен мономорфизм в соответствующую ортогональную группу

$$
\nu_{n}: W_{n} \rightarrow O_{n} .
$$

* Работа выполнена при финансовой поддержке РФФИ и DFG (гранты 96-01-00062G и RUS 113/190), а также INTAS. 
В. В. Вершинин, Э. Осса

Понятие группы кос было обобщено Э. Брискорном [23] для конечных групп Кокстера $W_{n}$ таким образом, что имеет место эпиморфизм, аналогичный эпиморфизму (1),

$$
\tau_{n}\left(W_{n}\right): B r\left(W_{n}\right) \rightarrow W_{n} .
$$

Предположим, что каноническое вложение евклидовых пространств $\mathbb{R}^{n} \subset \mathbb{R}^{n+1}$ индуцирует вложение соответствующих обобщенных групп кос $B r\left(W_{n}\right)<$ $B r\left(W_{n+1}\right)$, так что определена серия групп кос $B r\left(W_{n}\right), n=1,2, \ldots$ Примером могут служить серии групп кос типов $A_{n}, C_{n}$ и $D_{n}$. Им соответствуют следующие графы Кокстера:

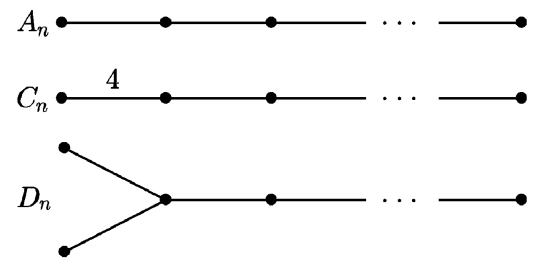

Из этих диаграмм видно, что группы $\operatorname{Br}\left(C_{n}\right)$ и $\operatorname{Br}\left(D_{n}\right)$ могут быть заданы образуюшими $w_{1}, \sigma_{1}, \ldots, \sigma_{n-2}$, такими, что элементы $\sigma_{i}$ удовлетворяют соотношениям группы кос и, кроме того, для группы $\operatorname{Br}\left(C_{n}\right)$ образующая $w_{1}$ коммутирует с $\sigma_{i}, i \geqslant 2$, и выполнено следующее соотношение:

$$
w_{1} \sigma_{1} w_{1} \sigma_{1}=\sigma_{1} w_{1} \sigma_{1} w_{1}
$$

а для группы $\operatorname{Br}\left(D_{n}\right)$ образующая $w_{1}$ коммутирует с $\sigma_{i}, i=1,3, \ldots, n-2$, и выполнено следующее соотношение:

$$
w_{1} \sigma_{2} w_{1}=\sigma_{2} w_{1} \sigma_{2} .
$$

Пусть $\left\{G_{n}\right\}$ - система подгрупп групп кос $\operatorname{Br}\left(W_{n}\right), n=1,2, \ldots$, такая, что $G_{n}$ есть подгруппа группы $G_{n+1}$, где обе группы рассматриваются как подгруппы группы $\mathrm{Br}\left(W_{n+1}\right)$. Это определяет композицию

$$
i_{n}: G_{n} \rightarrow \operatorname{Br}\left(W_{n}\right) \rightarrow W_{n} \rightarrow O_{n},
$$

такую, что диаграмма

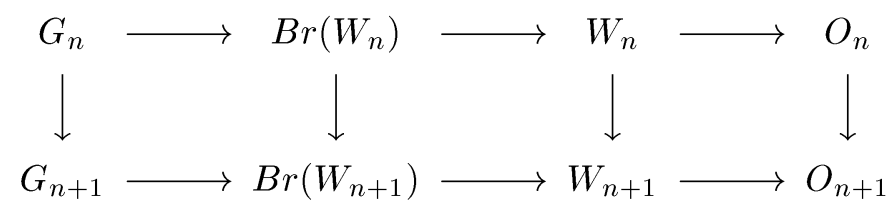

коммутативна. Предположим, что образ группы $G_{n}$ при композиции (2) лежит в группе вращений $S O_{n}$ для всех $n$. Тогда композиция (2) и коммутативная диаграмма (3) порождают отображения классифицирующих пространств и пространств Тома:

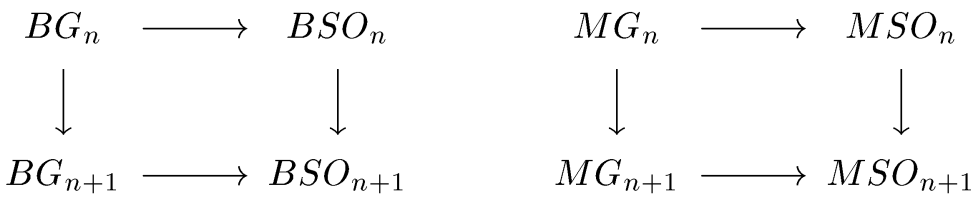


Пространства Тома $M G_{n}$ и $M S O_{n}$ и отображения $M G_{n} \rightarrow M S O_{n}$ порождают спектры Тома $M G$ и $M S O$ и морфизм спектров

$$
M G \rightarrow M S O .
$$

Пусть $F_{n}$ - моноид гомотопических самоэквивалентностей сферы $S^{n-1}$, a $S F_{n}$ - моноид ориентированных (степени 1 ) самоэквивалентностей сферы $S^{n-1}[24]$. Каноническое отображение

$$
\chi_{n}: S O_{n} \rightarrow S F_{n}
$$

индуцирует отображения классифицирующих пространств; следовательно, мы имеем композицию

$$
B G_{n} \rightarrow B S O_{n} \rightarrow B S F_{n} .
$$

Композиции (4) для всех $n$ индуцируют сферические расслоения над пространствами $B G_{n}$, которые, в свою очередь, порождают пространства Тома $M G_{n}$ и спектр Тома $M G$, так что спектр $M G$ можно считать порожденным композициями (4).

\section{§2. Гомологии коммутантов групп кос}

Пусть $G_{n}$ - это коммутант классической группы кос

$$
\left[B r_{n}, B r_{n}\right] \triangleleft B r_{n} .
$$

Заметим, что коммутанты групп $B r_{n}, B r\left(C_{n}\right)$ и $\operatorname{Br}\left(D_{n}\right)(n \geqslant 5)$ каиновы в терминологии В. Я. Лина [25], т. е. нет нетривиальных гомоморфизмов из них в абелевы группы [26]. Следовательно, плюс-конструкция (см., например, [27]) для них существует. Мы будем опускать индекс $\infty$ в обозначениях прямых пределов серий групп и писать просто $B r, B r(C)$ и $B r(D)$. Обозначим через $S^{3}\langle 3\rangle$ трехсвязное накрытие над $S^{3}$, которое определяется как гомотопический слой отображения $S^{3} \rightarrow K(\mathbb{Z}, 3)$, соответствующего образующей $H^{3}\left(S^{3}, \mathbb{Z}\right) . \Phi$. Коэн [18] доказал, что плюс-конструкция классифицирующего пространства коммутанта группы кос из бесконечного числа нитей $B r$ эквивалентна $\Omega^{2} S^{3}\langle 3\rangle$ - двукратному пространству петель над $S^{3}\langle 3\rangle$ :

$$
B[B r, B r]^{+} \cong \Omega^{2} S^{3}\langle 3\rangle
$$

и диаграмма

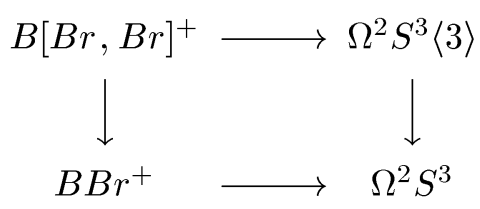

коммутативна. Рассмотрим теперь обобщенные группы кос серий $C_{n}$ и $D_{n}$. Когомологии этих групп были вычислены В. В. Горюновым $[15,16]$. Плюсконструкции классифицирующих пространств предельных групп этих серий эквивалентны соответственно $\Omega^{2} S^{3} \times \Omega S^{2}$ и $\Omega^{2} S^{3} \times S^{3}\{2\}$ (см. [10]), где $S^{3}\{2\}$ 
есть гомотопический слой отображения степени 2 из трехмерной сферы в себя

$$
S^{3} \rightarrow S^{3}
$$

Для обобщенных групп кос гомоморфизмы

$$
B r_{n} \rightarrow B r\left(C_{n+1}\right), \quad B r_{n} \rightarrow B r\left(D_{n+1}\right)
$$

соответствуют вложениям графов Кокстера. При гомоморфизме

$$
\rho_{C_{n+1}}: \operatorname{Br}\left(C_{n+1}\right) \rightarrow B r_{n}
$$

крайний левый образующий группы $\operatorname{Br}\left(C_{n+1}\right)$ переходит в единицу, а при гомоморфизме

$$
\rho_{D_{n+1}}: \operatorname{Br}\left(D_{n+1}\right) \rightarrow B r_{n}
$$

два крайних левых образующих группы $\operatorname{Br}\left(D_{n+1}\right)$ переходят в единственный крайний левый образующий группы $B r_{n}$. Очевидно, что соответствующие композиции равны тождественным гомоморфизмам группы $B r_{n}$.

ТЕОРЕмА 1. Для плюс-конструкиий классифииируюиих пространств коммутантов обобшенных арупп кос $\operatorname{Br}(C)$ и $\operatorname{Br}(D)$ имеют место эквивалентности

$$
\begin{aligned}
& B[B r(C), B r(C)]^{+} \cong \Omega^{2} S^{3}\langle 3\rangle \times \Omega S^{3}, \\
& B[B r(D), B r(D)]^{+} \cong \Omega^{2} S^{3}\langle 3\rangle \times S^{3}\{2\}
\end{aligned}
$$

такие, что канонические гомоморфизмьи $\mathrm{Br} \rightarrow \mathrm{Br}(C) \rightarrow \mathrm{Br} u \mathrm{Br} \rightarrow \mathrm{Br}(\mathrm{D})$ $\rightarrow B r$ порождают коммутативные диаграммы

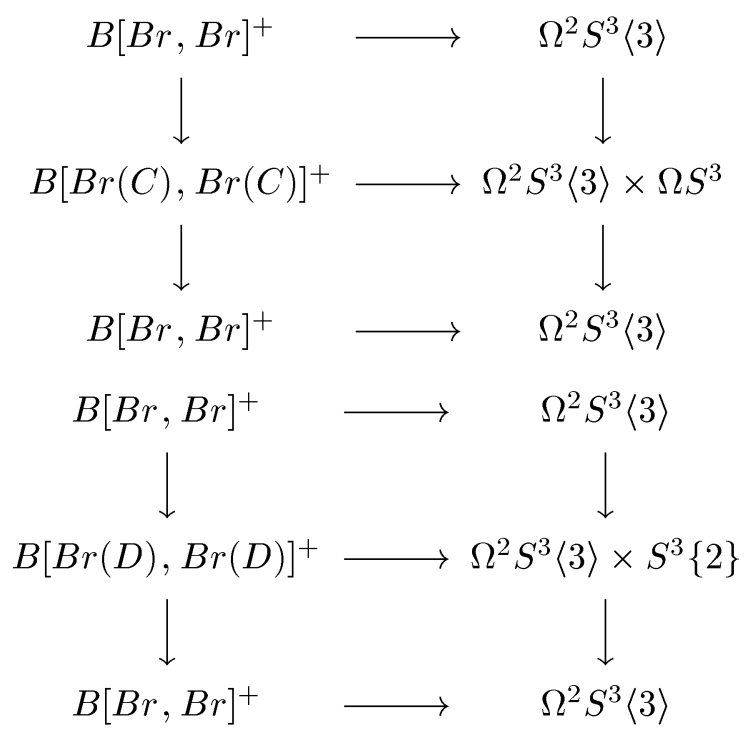

ДокАЗАТЕльСТво. Гомоморфизм $\rho$ порождает короткую точную последовательность

$$
e \rightarrow \operatorname{Ker} \rho_{C} \rightarrow \operatorname{Br}(C) \rightarrow B r \rightarrow e
$$


которая расщепляется справа. Абелеанизация группы $\operatorname{Br}(C)$ изоморфна $\mathbb{Z} \oplus \mathbb{Z}$. Поэтому имеет место точная последовательность групп

$$
e \rightarrow[\operatorname{Br}(C), \operatorname{Br}(C)] \rightarrow \operatorname{Br}(C) \rightarrow \mathbb{Z} \oplus \mathbb{Z} \rightarrow e,
$$

которая также расщепляется справа. Она соответствует точной последовательности для классической группы кос

$$
e \rightarrow[\mathrm{Br}, \mathrm{Br}] \rightarrow \mathrm{Br} \rightarrow \mathbb{Z} \rightarrow e,
$$

также расщепляемой. Пусть

$$
\rho_{C}^{\prime}:[B r(C), B r(C)] \rightarrow[B r, B r]
$$

— ограничение гомоморфизма $\rho_{C}$ на $[B r(C), B r(C)]$. Определим гомоморфизм

$$
\operatorname{deg}: \operatorname{Ker} \rho_{C} \rightarrow \mathbb{Z}
$$

как полную сумму степеней вхождений образующей $w_{1}$ при записи элемента $g \in \operatorname{Ker} \rho_{C}$, и пусть $K^{\prime}$ - его ядро. Точные последовательности, упоминаемые выше, порождают следуюшую коммутативную диаграмму:
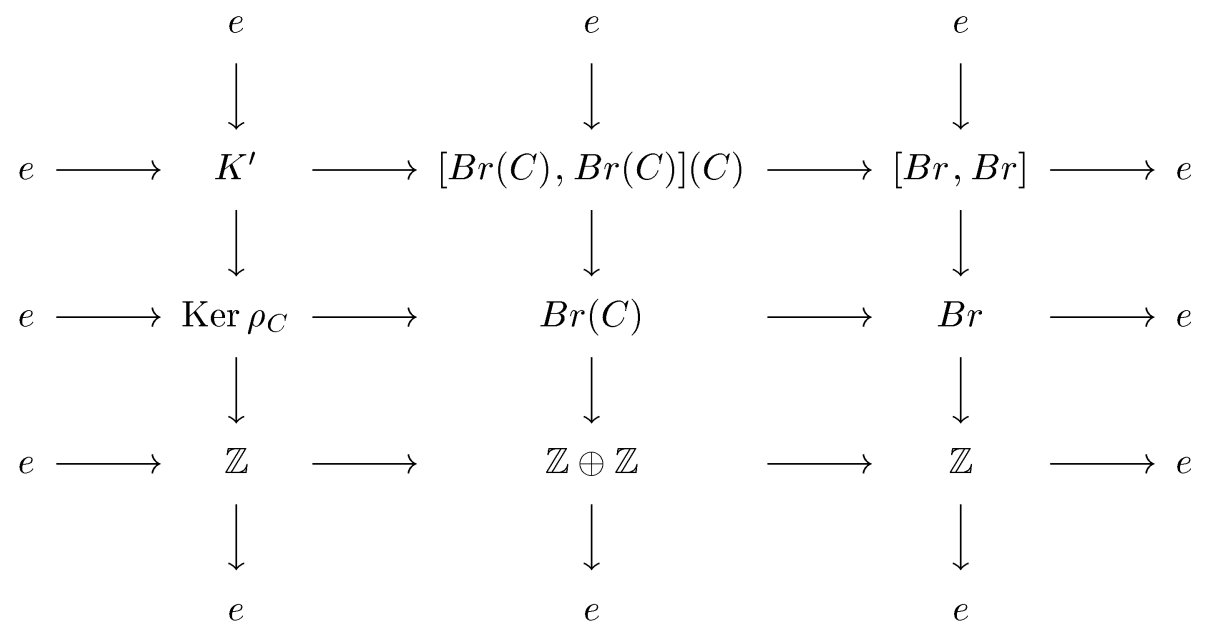

Используя тот факт, что последовательности (7)-(9) расщепляются, и диаграммный поиск, получаем, что верхняя строка в диаграмме (10) является расщепляемой справа точной последовательностью. Следовательно, классифицирующее пространство группы $[B r(C), B r(C)]$ эквивалентно декартову произведению классифицирующих пространств групп $[B r, B r]$ и $K^{\prime}$. Значит, плюс-конструкция $B[B r(C), B r(C)]$ равна $\Omega^{2} S^{3}\langle 3\rangle \times \Omega S^{3}$ и теорема доказана для группы типа $C$. Для группы $B r(D)$ доказательство аналогично.

\section{§3. Спектры Тома}

Спектр Тома над $\Omega^{2} S^{3}\langle 3\rangle$, соответствующий коммутативной диаграмме

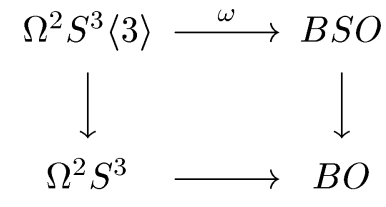


изучался Маховальдом [6], а также Коэном, Мэем и Тейлором [28]. Было доказано, что спектр $M \omega$ после локализации вне 2 эквивалентен спектру, определяюшему обычные гомологии с коэффициентами в локальном кольце $\mathbb{Z}_{(2)}$. Используя стабильное расщепление Снэйта [29] пространства $\Omega^{2} S^{3}$ и тот факт, что $B S F$ является бесконечнократным пространством петель, Маховальд [6] строит отображение

$$
h: \Omega^{2} S^{3} \rightarrow B S F
$$

и с его помощью отображение

$$
\begin{aligned}
g: \Omega^{2} S^{3}\langle 3\rangle \stackrel{\Delta}{\longrightarrow} \Omega^{2} S^{3}\langle 3\rangle \times \Omega^{2} S^{3}\langle 3\rangle & \\
& \rightarrow \Omega^{2} S^{3} \times \Omega^{2} S^{3}\langle 3\rangle \stackrel{h \times B \chi \circ \omega}{\longrightarrow} B S F \times B S F \rightarrow B S F,
\end{aligned}
$$

такое, что спектр Тома, полученный из сферических расслоений с помощью $g$, является в точности спектром $H \mathbb{Z}$ (без локализации).

Гомоморфизм

$$
i:[\mathrm{Br}, \mathrm{Br}] \rightarrow S O
$$

индуцирует спектр Тома, который мы будем обозначать через $M[B r, B r]$ и который эквивалентен $H \mathbb{Z}_{(2)}$ после локализации вне 2. Группы $\left[\operatorname{Br}\left(C_{n}\right)\right.$, $\left.\operatorname{Br}\left(C_{n}\right)\right]$ и $\left[\operatorname{Br}\left(D_{n}\right), \operatorname{Br}\left(D_{n}\right)\right]$ канонически вложены в группу $S O_{n}$. Рассмотрим спектры Тома, индуцированные этими вложениями.

ТЕОРема 2. Канонические гомоморфизми

$$
\left[\mathrm{Br}\left(C_{n}\right), \mathrm{Br}\left(C_{n}\right)\right] \rightarrow S O_{n}, \quad\left[\mathrm{Br}\left(D_{n}\right), \mathrm{Br}\left(D_{n}\right)\right] \rightarrow S O_{n}
$$

порождают спектры Тома $M[B r(C), B r(C)]$ и $M[B r(D), B r(D)]$, которые после локализачии вне 2 эквивалентны следующим букетам:

$$
\begin{aligned}
& M[\operatorname{Br}(C), B r(C)]_{(2)} \cong \bigvee_{l=0}^{\infty} S^{2 l} H \mathbb{Z}_{(2)}, \\
& M[\operatorname{Br}(D), B r(D)]_{(2)} \cong H \mathbb{Z}_{(2)} \vee\left(\bigvee_{l=1}^{\infty} S^{2 l} H \mathbb{Z} / 2\right) .
\end{aligned}
$$

Спектр $M[B r(C), B r(C)]$ мультипликативен и его кольчо коэффичиентов (после локализачии) изоморфно кольчу многочленов

$$
\mathbb{Z}_{(2)}[y], \quad \operatorname{deg} y=2 .
$$

ДокАЗАТЕЛЬСтво. Рассмотрим вложения

$$
\left[\operatorname{Br}\left(C_{m}\right), B r\left(C_{m}\right)\right] \times\left[B r\left(C_{n}\right), B r\left(C_{n}\right)\right] \rightarrow B r\left(C_{m}\right) \times B r\left(C_{n}\right)
$$

и спаривание для обобщенных групп кос типа $C$, изучавшееся в [30],

$$
\mu_{m, n}: \operatorname{Br}\left(C_{m}\right) \times \operatorname{Br}\left(C_{n}\right) \rightarrow B r\left(C_{m+n}\right) .
$$

Композиция этих гомоморфизмов задает спаривание для коммутантов

$$
\mu_{m, n}:\left[B r\left(C_{m}\right), B r\left(C_{m}\right)\right] \times\left[B r\left(C_{n}\right), B r\left(C_{n}\right)\right] \rightarrow\left[B r\left(C_{m+n}\right), B r\left(C_{m+n}\right)\right] .
$$


Это спаривание делает спектр $M[B r(C), B r(C)]$ мультипликативным.

Коммутативные диаграммы (5) и (6) порождают коммутативные диаграммы спектров Тома

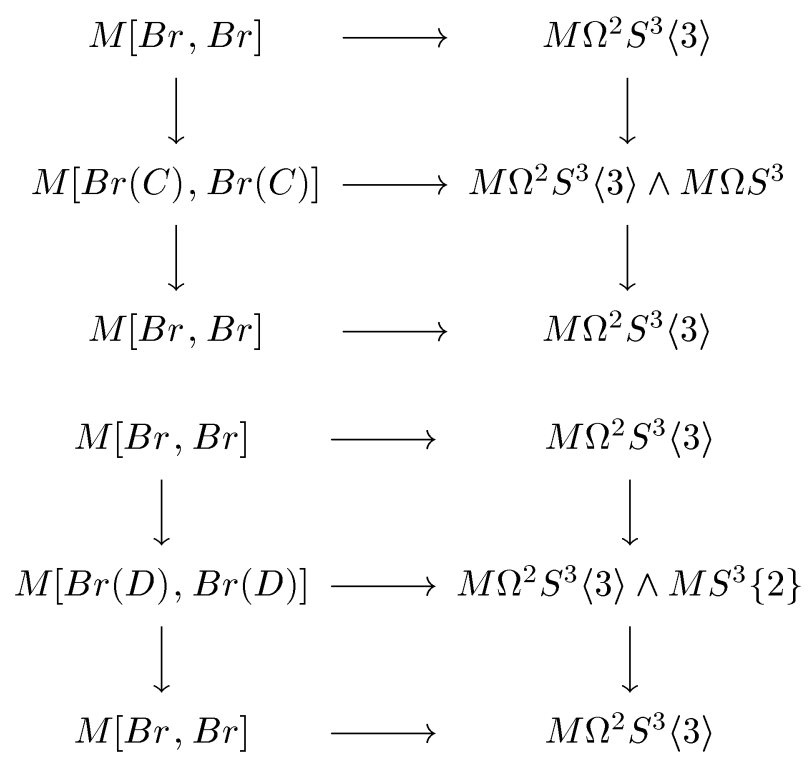

где горизонтальные стрелки являются гомотопическими эквивалентностями. Рассмотрим спектры, локализованные вне 2. Тогда

$$
\begin{aligned}
& M[B r(C), B r(C)]_{(2)} \cong H \mathbb{Z}_{(2)} \wedge M \Omega S^{3}, \\
& M[B r(D), B r(D)]_{(2)} \cong H \mathbb{Z}_{(2)} \wedge M S^{3}\{2\}
\end{aligned}
$$

Следовательно, гомотопическими группами спектров $M[\operatorname{Br}(C), \operatorname{Br}(C)]_{(2)}$ и $M[B r(D), B r(D)]_{(2)}$ являются группы гомологий спектров $M \Omega S^{3}$ и $M S^{3}\{2\}$ с коэффициентами в $\mathbb{Z}_{(2)}$ :

$$
\begin{aligned}
& \pi_{*}\left(M[B r(C), B r(C)]_{(2)}\right)=H_{*}\left(M \Omega S^{3} ; \mathbb{Z}_{(2)}\right), \\
& \pi_{*}\left(M[B r(D), B r(D)]_{(2)}\right)=H_{*}\left(M S^{3}\{2\} ; \mathbb{Z}_{(2)}\right)
\end{aligned}
$$

В первом случае эти гомологии изоморфны кольцу многочленов над $\mathbb{Z}_{(2)}$ от образующей степени 2 :

$$
H_{*}\left(M \Omega S^{3} ; \mathbb{Z}_{(2)}\right) \cong \mathbb{Z}_{(2)}[y], \quad \operatorname{deg} y=2 .
$$

Во втором случае имеем

$$
H_{i}\left(M S^{3}\{2\} ; \mathbb{Z}_{(2)}\right)= \begin{cases}\mathbb{Z}_{(2)} & \text { для } i=0, \\ \mathbb{Z} / 2 & \text { для } i=2 k, k>0, \\ 0 & \text { для } i \neq 0,2 k .\end{cases}
$$


Используя эти факты и диаграммы (12) и (13), мы строим отображения

$$
\begin{gathered}
\bigvee_{l=0}^{\infty} S^{2 l} H \mathbb{Z}_{(2)} \rightarrow M[B r(C), \operatorname{Br}(C)]_{(2)}, \\
M[B r(D), B r(D)]_{(2)} \rightarrow H \mathbb{Z}_{(2)} \vee\left(\bigvee_{l=1}^{\infty} S^{2 l} H \mathbb{Z} / 2\right),
\end{gathered}
$$

которые являются гомотопическими эквивалентностями.

Канонические мономорфизмы $[\operatorname{Br}(C), B r(C)] \rightarrow B r(C)$ и $[\operatorname{Br}(D), B r(D)]$ $\rightarrow B r(D)$ индуцируют морфизмы спектров Тома. Из описания спектров Тома для $\operatorname{Br}(C)$ и $\operatorname{Br}(D)$ [30] мы видим, что для серии $C$ имеется морфизм мультипликативных спектров, такой, что для колец коэффициентов он дает кольцевой гомоморфизм

$$
\mathbb{Z}_{(2)}[y] \rightarrow \mathbb{Z} / 2[s]
$$

определенный тем, что $y$ отображается в $s^{2}$. Для серии $D$ имеется эквивалентность между слагаемыми вида $H \mathbb{Z} / 2$ в положительных размерностях спектра $M[B r(D), B r(D)]_{(2)}$ и соответствующими слагаемыми спектра $M B r(D)$.

ЗАМЕЧАНИЕ. Если В КОМПОЗИцияХ

$$
\begin{aligned}
& B[B r(C), B r(C)] \rightarrow \Omega^{2} S^{3}\langle 3\rangle \times \Omega S^{3} \rightarrow B S O \rightarrow B S F \\
& B[B r(D), B r(D)] \rightarrow \Omega^{2} S^{3}\langle 3\rangle \times S^{3}\{2\} \rightarrow B S O \rightarrow B S F
\end{aligned}
$$

мы заменим отображение $\Omega^{2} S^{3}\langle 3\rangle \rightarrow B S O \rightarrow B S F$ на отображение $g$ из (11), мы получим спектры Тома, в которых слагаемые вида $H \mathbb{Z}_{(2)}$ заменены на $H \mathbb{Z}$.

Эта работа была выполнена, главным образом, во время посещения первым автором университета г. Вупперталь (ФРГ). Он выражает благодарность математическому отделению этого университета и работающей там группе топологов за гостеприимство и замечательные условия для работы.

\section{ЛИтЕРАТУРА}

1. Арнольд В. И. О некоторых топологических инвариантах алгебраических функций. Труды Моск. матем. о-ва, 21, 27-46 (1970).

2. Арнольд В. И. Топологические инварианты алгебраических функций. II. Функц. анализ и его прил., 4, вып. 2, 1-9 (1970).

3. Васильев B. A. Когомологии групп кос и сложность алгоритмов. Функц. анализ и его прил., 22, вып. 3, 15-24 (1988).

4. Smale $S$. On the topology of algorithms. I. J. Complexity, 4, 81-89 (1987).

5. Mahowald $M$. A new family in $\pi_{*}^{s}$. Topology, 16, 249-254 (1977).

6. Mahowald M. Ring spectra which are Thom complexes. Duke Math. J., 46, No. 3, 549-259 (1977).

7. Cohen F. R. Braid orientations and bundles with flat connections. Invent. Math., 46, 99-110 (1978).

8. Deligne $P$. Les immeubles des groups de tresses généralisés. Invent. Math., 17, 273-302 (1972). 
9. Segal $G$. Configuration spaces and iterated loop spaces. Invent. Math., 21, 213-221 (1973).

10. Фукс Д. Б. Квилленизация и бордизмы. Функц. анализ и его прил., 8, вып. 1, 36-42 (1974).

11. Cohen F. R., Lada T., May J. P. The homology of iterated loop spaces. Lect. Notes in Math., Vol. 533, Springer-Verlag, Berlin, 1976.

12. Ossa E. On the cohomology of configuration spaces. In: Algebraic Topology: New Trends in Localization and Periodicity. Barcelona Conference on Algebraic Topology, 1994, Birkhäuser, Basel, 1996, pp. 353-361.

13. Фукс Д. Б. Когомологии групп кос $\bmod 2$. Функц. анализ и его прил., 4, вып. 2, 62-75 (1970).

14. Вайнштейн Ф. В. Когомологии групп кос. Функц. анализ и его прил., 12, вып. 2, 72-73 (1978).

15. Горюнов B. B. Когомологии групп кос серий $C$ и $D$ и некоторые стратификации. Функц. анализ и его прил., 12, вып. 2, 76-77 (1978).

16. Горюнов B. B. Когомологии групп кос серий $C$ и $D$. Труды Моск. матем. о-ва, 42, 234-242 (1981).

17. Арнольд В. И. Кольцо когомологий группы крашеных кос. Матем. заметки, 5, № 2, 227-231 (1969).

18. Cohen F. R. Artin's braid groups and classical homotopy theory. Contemp. Math., 44, 207-226 (1985).

19. Френкель Э. В. Когомологии коммутанта группы кос. Функц. анализ и его прил., 22, вып. 3, 91-92 (1988).

20. Artin E. Theory of braids. Ann. of Math., 48, No. 1, 101-126 (1947).

21. Birman J. Braids, links, and mapping class groups. Ann. Math. Stud., Vol. 82, 1974.

22. Бурбаки H. Группы и алгебры Ли. Мир, М., 1972.

23. Brieskorn E. Sur les groupes de tresses. Séminaire Bourbaki, 1971/72, exp. 401; Lect. Notes in Math., Vol. 317, Springer-Verlag, Berlin a.o., 1973, pp. 21-44. [Имеется перевод: Брискорн Э. О группах кос (по В. И. Арнольду), Математика, 18, вып. 3, 46-59 (1974).]

24. Stasheff J. A classification theorem for fiber spaces. Topology, 2, 239-246 (1963).

25. Лин В. Я. Косы Артина и связанные с ними группы и пространства. Итоги науки и техники (Алгебра, топология, геометрия), т. 17, 1979, с. 159-227.

26. Зинде В. М. Коммутанты групп Артина. УМН, 30, вып. 5, 207-208 (1975).

27. Адамс Дж. Бесконечнократные пространства петель. Мир, М., 1982.

28. Cohen F. R., May J. P., Taylor L. R. $K(Z, 0)$ and $K\left(Z_{2}, 0\right)$ as Thom spectra. Illinois J. Math., 25, No. 1, 99-106 (1981).

29. Snaith V. P. A stable decomposition of $\Omega^{n} S^{n} X$. J. London Math. Soc. (2), 7, $577-583$ (1974).

30. Vershinin $V$. $V$. Thom spectra of generalized braid groups. Preprint No. 95/02-2, Université de Nantes, 1995.

Институт математики СО РАН, Новосибирск

Поступило в редакцию e-mail: versh@math.nsc.ru 30 июля 1997 г.

Fachbereich Mathematik, Univ. Wuppertal, Germany

e-mail: ossa@math.uni-wuppertal.de 